\title{
Lumbar spine loading during dressage riding
}

\section{Abstract}

5 Context: Lower back pain is prevalent in horse riders as a result of the absorption of repetitive

6 and multi-planar propulsive forces from the horse. GPS technology provides potential for in-

7 vivo measurement of planar loading during riding. Objective: To quantify the uni-axial

8 loading at the lumbar and cervico-thoracic spine during dressage elements. Design: Repeated

9 measures, randomized order. Setting: Equestrian arena. Patients (or Other Participants):

1021 female dressage riders. Intervention(s): Each rider completed walk, rising trot, sitting trot and canter trials in randomized order. A GPS unit was placed within customized garments at C7 and L5, collecting tri-axial accelerometry data at 100Hz. Outcome Measures: PlayerLoad based on the rate of change of acceleration, and calculated in the anteroposterior (AP), mediolateral (ML) and vertical planes during each trial. Results: There was no significant main effect for GPS location in the AP $(P=0.758), \operatorname{ML}(P=0.876)$ or $\mathrm{V}(P=0.762)$ planes. There was a significant main effect for pace in all trials $(P<0.001)$, with successive elements eliciting significantly greater loading $(P \leq 0.030)$ in all planes in the order walk $<$ rising trot $<$ canter $<$ sitting trot. There was a significant placement $x$ element interaction only in the AP plane $(P=0.032)$ with AP loading greater at L5 during walk, rising trot and canter trials, but greater at C7 during sitting trot. Conclusions: The significant main effect for dressage element was indicative of greater pace of the horse, with faster pace activities eliciting greater loading in all planes. In-vivo measurement of spinal accelerometry has application in the objective measurement and subsequent management of lumbar load for riders. 
27 Horseback riding has been highlighted as one of the most dangerous recreational sports. ${ }^{1}$ Of particular concern is low back pain with $73 \%$ of riders reporting a significantly higher incidence than the general population. ${ }^{2}$ It has been reported that $74 \%$ of elite dressage riders competed whilst experiencing pain, and for $76 \%$ of riders this pain was in the lower back. ${ }^{3}$ Kraft et al. (2009) postulated that the cause of low back pain in riders might be an overuse syndrome of the lumbar spine ${ }^{4}$ as a result of the repetitive compressive, torsional and bending loads absorbed by the rider. ${ }^{5}$ The authors used magnetic resonance imaging to investigate associations between low back pain, but this might be considered a relatively inaccessible means of managing load towards an overuse injury paradigm. The forces experienced by the rider are largely translated from the horse,${ }^{6}$ via the strirrup, through the lower limbs and ultimately to the spine of the rider. Torque transducers embedded within the stirrup of a riding simulator offer insight into the forces transmitted from the horse, ${ }^{7}$ and laboratory-based biomechanical models using a combination of kinematic and kinetic data provide potential to quantify segmental forces,${ }^{8}$ but these methods offer limited ecological validity.

Contemporary means of quantifying lumbar spine loading is provided by applications in global positioning satellite (GPS) technology, which enable measurement in the clinical or sporting context. Typical GPS analysis metrics include distance and velocity profiling, based on derivations of changes in location, but embedded tri-axial accelerometers facilitate the measurement of mechanical loading in-vivo. The tri-axial function and relatively high sampling frequency at $100 \mathrm{~Hz}$ has enabled investigation of lumbar loading in cricket ${ }^{9}$ where lumbar injuries are a primary concern. Whilst the GPS unit is typically positioned at C7 in a sporting context to facilitate satellite reception, in a more controlled clinical context the unit has been repositioned to provide greater anatomical relevance to the injury concern. ${ }^{9,10}$ With direct relevance to a focus on low back pain in riders, differences in planar loading at the 
51 lumbar and cervico-thoracic spine in cricket bowling have been associated with injury epidemiology, ${ }^{9}$ and subsequently used to consider alternate workload management strategies in young bowlers. ${ }^{11}$

54 The magnitude of force transmitted from the horse is likely to be influenced by the pace of the 55 horse, ${ }^{12,13}$ and thus pace is a fundamental consideration in the present study. The dressage discipline of horse riding demands moderation of pace whilst maintaining postural poise, and thus provides a choreographed routine of varying pace across elements classified as walk, rising trot, sitting trot and canter. Furthermore, Kraft et al. (2009) reported that it was only in the dressage discipline where riders exhibited signal alterations of the lumbar disks, attributed to the greater physical demands and intensity of daily training for a dressage rider. ${ }^{4}$ The authors suggested that the intensive training in a seated position across all paces might be the cause of lumbar spine damage by repetitive microtrauma and recommended further research in the dressage discipline. ${ }^{4}$ Lewis and Kennerley (2017) in considering the incidence of elite riders competing with lower back pain more specifically advocated research into appropriate management techniques, reporting that over half of riders used over the counter medication in an attempt to relieve pain.

The aim of our study was to quantify the influence of pace on planar loading at the lumbar and cervico-thoracic spine during riding. The ecological validity afforded by the in-vivo measure of acceleration at the spine might offer clinical applications in developing load management strategies for riders.

\section{Methods}

\section{Design}

74 This was a repeated-measures field-based study, with experimental trials (relating to dressage element) completed in a randomized order. The study was conducted in the equestrian arena 
commonly used by each rider, with the rider using their own horse and dressage saddle.

77 Familiarisation trials were completed to ensure that the choreographed activity profile for each element was habitual and common to all horse-rider pairs. The independent variables were the location of the GPS unit for quantifying loading, and the dressage element. The dependent variables were the uni-axial PlayerLoad in each of the anteroposterior (AP), mediolateral (ML) and vertical (V) planes of movement.

\section{Participants}

21 female dressage riders were recruited from training sessions and clinics run by British

Dressage trainers and coaches. All participants were required to be free from musculoskeletal injury for at least six months prior to testing. Inclusion criteria also required that participants were aged 18 years and above with at least 3 years riding experience, to ensure appropriate musculoskeletal physiology and functional anatomy maturation and adaptation to the sport. ${ }^{13}$ Riding experience has also been identified as a risk factor for injury in dressage riders and so a minimum 100 hours of experience was established as an inclusion criteria. ${ }^{14}$ All riders had competed at British Dressage events ranging from Preliminary competitions to Grand Prix events. All participants provided written consent, and the study was granted ethical approval by the Departmental research ethics committee, in accord with the spirit of the Helsinki

\section{Declaration.}

\section{$94 \quad$ Procedures}

95 All participants completed familiarisation trials on their own horse and within the same arena to ensure familiarity with the choreographed routine devised for each technical element. The arena measured $60 \mathrm{~m} \times 20 \mathrm{~m}$ with a rubber and sand surface, standardized across all trials. The horse was self-selected by the rider, irrespective of age, breeding or conformity of the horse. The saddle was also self-selected by the rider, with the requirement that all saddles were dressage-specific. During the experimental trial a standardised warm-up was completed which 
included all test elements and each rider wore their own riding clothes in accordance with regulation and to ensure comfort. A neoprene vest and belt were also worn to house the GPS unit (Catapult Minimaxx S4, Catapult Innovations, Victoria, Australia), located at C7 and L5 respectively as shown in Figure 1, and secured with athletic tape.

Each participant completed experimental trials of the following dressage elements within a single experimental session: walk, rising trot, canter, sitting trot. The order in which the elements were completed was randomized, but the duration of each element was consistent. A total duration of 60 seconds was used for each element, with a 20 second analysis window selected to avoid transition in acceleration or deceleration, and to ensure steady-state was achieved. Trials of each element were completed for both a left rein and a right rein to acknowledge the potentially confounding variable of dominance/preference, with all elements completed in a circular riding pattern. No lateral movements were included, reflecting the relative experience and level of these riders.

117 The tri-axial accelerometer embedded within the GPS unit collected acceleration data at 100Hz. This data was used to calculate PlayerLoad in each of the anteroposterior (AP), mediolateral (ML) and vertical (V) movement planes. PlayerLoad is defined by the rate of 120 change of acceleration, with PlayerLoad quantified at C7 and L5 as described by Greig and 121 Nagy (2017).

\section{Statistical Analyses}

123 Data are presented as mean \pm standard deviation in the subsequent section. A univariate 124 general linear model was used to investigate a main effect for GPS location (C7, L5), a main 125 effect for dressage element (walk, rising trot, canter, sitting trot), and a placement $x$ element 
interaction for PlayerLoad in each plane. Where appropriate, post-hoc analysis was performed

127 to identify differences between locations or elements. Data was checked for normality a priori,

128 using histograms, q-q plots, skewness and kurtosis, and a Shapiro-Wilk test. Mauchly's test of

129 Sphericity was performed for the dependent variables, with a Greenhouse Geisser correction

130 included if test significance was indicated. Statistical significance was set at $P<0.05$, and partial eta squared $\left(\eta^{2}\right)$ is reported as a measure of effect size.

133 Results

134 Preliminary analysis revealed no significant main effect for rein (clockwise vs counter135 clockwise direction of riding) on PlayerLoad in the AP $\left(P=0.447 ; \eta^{2}=0.002\right), \operatorname{ML}(P=0.838$;

$\left.136 \eta^{2}<0.001\right)$ or $\mathrm{V}\left(P=0.423 ; \eta^{2}=0.002\right)$ planes. Furthermore, no rein x element $(P \geq 0.808)$,

137 rein $\mathrm{x}$ GPS location $(P \geq 0.398)$, or rein $\mathrm{x}$ element $\mathrm{x}$ location $(P \geq 0.907)$ interactions were observed. Therefore, in subsequent analyses the data was pooled for rein.

139 Figure 2 summarises the influence of GPS location and dressage element on the planar loading 140 response.

143 There was no significant main effect for GPS placement in the AP $(P=0.758)$, ML $(P=$ $1440.875)$ or $\mathrm{V}(P=0.762)$ planes $\left(\eta^{2}<0.001\right)$.

145 There was a significant main effect for element in all planes $\left(P<0.001 ; \eta^{2} \geq 0.488\right)$. In the AP 146 plane the loading increased significantly $(P \leq 0.030)$ at each progression from walk to rising 147 trot, to canter, to sitting trot. This same pattern was evident in the ML $(P \leq 0.021)$ and V $(P$ $148 \leq 0.029)$ planes, with the same hierarchical ordering of elements, indicative of the increased 149 pace. 
150 There was no significant GPS location $x$ dressage element interaction in the ML $(P=0.125$;

$\left.151 \eta^{2}=0.017\right)$ or $\mathrm{V}\left(P=0.351 ; \eta^{2}=0.010\right)$ planes, but there was a significant interaction $(P=$

$\left.1520.032 ; \eta^{2}=0.026\right)$ in AP loading. This interaction was identified as greater AP loading at C7

153 during the sitting trot, but greater AP loading at L5 during the walk, rising trot and canter

154 trials.

155 Figure 3 summarises the relative planar contributions to total PlayerLoad, defined as the sum 156 of the three axial planes. Across all trials the relative contributions of AP:ML:V loading was

157 27:18:55.

158

159

**Insert Figure 3 near here**

160

161 There was no significant main effect for unit location in the relative contributions to loading

162 from the $\operatorname{AP}\left(P=0.121 ; \eta^{2}=0.015\right), \operatorname{ML}\left(P=0.581 ; \eta^{2}=0.002\right)$, or $\mathrm{V}\left(P=0.313 ; \eta^{2}=0.006\right)$ 163 planes.

164 There was no main effect for riding element in the AP contributions to loading $\left(P=0.323 ; \eta^{2}\right.$ $165=0.021$, with AP contribution maintained at $26.8 \pm 4.2 \%$ across all trials. There was a 166 significant main effect for element in both the ML $\left(P<0.001 ; \eta^{2}=0.592\right)$ and V $(P<0.001$;

$\left.167 \eta^{2}=0.433\right)$ planes. There was significantly lower $\mathrm{V}$ contribution in walking than in all other 168 activities $(P<0.001)$, with a compensatory increase in ML loading during walking $(P<0.001)$

169 than all other activities (which were themselves not different).

170 In relative loading there was no significant location $\mathrm{x}$ element interaction in any plane $(P \geq$ 171 $\left.0.293 ; \eta^{2} \leq 0.023\right)$

172

173 Discussion 
174 The aim of the present study was to examine the influence of pace on the planar loading of the 175 spine during dressage elements, given the prevalence of low back pain in riders. ${ }^{3,4}$ The high

176 incidence of elite dressage riders competing with lower back pain coupled with the longevity 177 of a rider's career highlights the clinical implications for the study. ${ }^{3}$ To reflect contemporary 178 applications in sport-specific injury paradigms, accelerometers were placed at the lumbar and 179 cervico-thoracic spine..$^{9,11}$ The spine has distinct sagittal plane curvatures that facilitate the 180 absorption and transmission of load, ${ }^{15}$ but there was no difference between L5 and C7 in either 181 the magnitude of planar load or the relative contributions of each plane to total load. This 182 suggests that no additional absorption of force (from the horse via the stirrup and lower limbs) 183 is achieved by the spine. Good postural control is required to enable the rider to sit correctly 184 in the face of perturbations from the horse. ${ }^{16}$ The rider must maintain a correct and balanced 185 posture whilst also responding to the horse's motion, stabilising their upper body and accommodating perturbations from the horse. ${ }^{17}$ Experienced dressage riders employ a 187 combination of posterior pelvic and anterior trunk tilting to flatten the lumbar curvature, ${ }^{8}$ and 188 use feed forward mechanisms to anticipate the horse's movements and compensate for 189 perturbations from the horse. ${ }^{18}$ Upper kinetic chain adaptations might also influence the 190 apparent lack of load dissipation through the spine, with dressage riders manipulating trunk 191 position for horse control and pace control to a larger extent than show jumpers and other riding 192 disciplines. ${ }^{4}$ Increased anterior rotation of the upper body relative to the pelvis has been 193 observed as trotting speed increases, ${ }^{19}$ with movements of the trunk and head considered 194 pivotal to riding effectiveness ${ }^{17}$ and with additional work at the reins to control the horse.

195 At sitting trot the rider is subjected to repetitive impacts with the saddle and as each of the 196 horse's feet hit the ground in diagonal pairs the rider is jolted in all planes. To avoid being 197 unbalanced at each diagonal stride the rider needs to activate their core and be flexible at the 198 hips in an effort to reduce the lumbar load. Thrasher et al. (2010) studied the responses of the 
trunk to multidirectional perturbations during unsupported sitting and described significantly greater displacement in response to diagonal (rather than linear) perturbations, ${ }^{20}$ characteristic of trotting and with implications for the influence of horse gait and pace on spinal loading. This is supported by the location $\mathrm{x}$ element interaction in the anteroposterior plane. Whilst AP 203 loading was greater at L5 during the walk, rising trot and canter trials, the sitting trot was associated with greater AP load at C7. In contrast to the four-beat gait at walk, trot is a twobeat gait where the horse moves its legs in diagonal pairs in unison. A trot can be hard for the rider to sit to because the horse drops between beats and bounces up when the next pair of legs strike the ground. At this point the rider can be jolted up and out of the saddle and hit the horse with considerable force on the way back down. Differences in trunk and thigh angle between impacts of the horse's diagonal limb pairs have been reported during trot which were not apparent at canter, ${ }^{21}$ highlighting postural changes between limb impacts in trot. At sitting trot all the forces pass through the seat whereas in rising trot the rider stands up and down from the knees, rising up and down with the horse rather than impacting with the horse at each stride.

213 Whilst greater force at the stirrups has been observed in the rising trot, ${ }^{22}$ saddle forces are 214 greater at sitting trot. ${ }^{23}$ With greater PlayerLoad exhibited in the sitting trot in the present study, the greater AP loading might reflect compensations from the upper kinetic chain to tolerate this demanding seated position, and an alternate means of attenuating impact.

217 Loading in each plane was significantly influenced by the pace of the horse, increasing 218 successively in the order walk to rising trot to canter. This supports previous research that identified the force acting on the horses back increased with riding pace. ${ }^{12}$ Walk was consistently associated with lowest loads in each plane and is the slowest of the paces as the horse moves in a 4-beat rhythm following the sequence: left hind leg, left front leg, right hind 222 leg, right front leg. The horse will alternate between having 3 or 2 feet on the ground, unlike 223 the other paces which have only 2 feet (trot) or 1 foot (canter) on the ground. Trot is a two- 
beat gait characterised by diagonal pairs and has a wide variation in speeds, whilst canter is a more complex three beat gait. At canter the horse uses a single hind leg to propel itself forward, prior to the next beat where the horse catches itself on the opposite hind leg and both front legs, and a final beat where the horse catches itself on one front leg (opposite to the propelling hind leg). Sitting trot elicited the greatest loading in all planes, indicative of the previous discussion regarding impact with the horse by maintaining the seated position as the horse follows a naturally rhythmic progression. At sitting trot the forces exerted on the rider are not unidirectional, are hard to predict and change rapidly making maintenance of the seated equilibrium much harder. At rising trot the rider moves up and down out of the saddle and is not in the saddle long enough to be exposed to the repetitive impacts experienced at sitting trot. The rhythm is easier to predict than at sitting trot allowing time for the active subsystem of core muscles to provide dynamic stabilization of the spine and attenuate transmission of forces up the spine. ${ }^{24,25}$ Canter is also more gentle and predictable rhythm to sit to than trot and elicited a lower loading magnitude than sitting trot.

Relative planar contributions to loading revealed an overall ratio of 27:18:55 in the AP:ML:V axes. This reflects the forward direction of travel and the rhythmic rise and fall of the horse, particularly at the higher speeds. At the slower walking pace there was significantly lower vertical contributions to loading, and Kraft et al. (2007) highlighted that for dressage riders with pre-existing back pain the pace "walk" seemed to have a positive influence on pain intensity whilst riding. ${ }^{2}$ There was a subsequent compensatory increase in the relative mediolateral contributions with anteroposterior contribution consistent across all elements and indicative of the maintenance in posture on the horse. The mediolateral contributions to loading most likely reflect the compensatory movements in response to the movement of the horse. For example, when the horse is propelling itself forward in canter with its left hind leg (leads with right foreleg) the rider's pelvis is lower on the right, and the asymmetry in the horse 
249 gait is likely to transmit to kinematic compensations at the spine. Of note there was no influence of rein on loading, and no interaction with accelerometer placement or pace,

251 suggesting no impact of any potential bilateral preference in the sample of horse-rider pairs. ${ }^{26}$

252 This non-invasive method of quantifying load in an equestrian context with high ecological

253 validity might be extended to consider loading at the saddle or as an alternate means of assessing horse gait kinematics. In this study the rider self-selected their own horse and dressage saddle to facilitate horse-rider familiarity. However future research might consider the influence of horse age, breed, performance level and conformity on loading magnitudes. Saddle fit might also influence loading magnitudes, and this experimental method might help to inform saddle design for both horse and rider. ${ }^{27}$ There is also value in a comparison of different arena surfaces for dressage riders which has been considered in relation to the risk of injury to the horse. ${ }^{28}$ The present study standardised arena size and surface, but the impact of different surfaces on loading is worthy of investigation. The size of the arena will also dictate riding patterns, with all elements in this study performed using a riding circle and negating any lateral movements. With an increased performance level of horse and rider the range and complexity of technical skills might be expanded. The same methodological paradigm might also be applied to other equestrian disciplines, and to more closely examine associations between spinal loading and movement screen disorders. Further research might consider associations between spinal loading and low back pain prevalence, anthropometric and postural analysis of the rider, spine and hip flexibility, etc.

\section{Conclusion}

271 The prevalence of lower back pain in dressage riders is a primary concern, and GPS technology

272 provides an in-vivo method for quantifying lumbar spine loading whilst riding. Loading was not sensitive to rein, or to location of the tri-axial accelerometer with placement therefore 
274 advocated at the closest proximity to the injury site. Loading was sensitive to pace of the horse,

275 with sitting trot highlighted as eliciting the greatest load. The relative planar contributions to

276 loading were consistent across all paces, and thus a consideration of magnitude suggests that

277 sitting trot is the pace requiring greatest moderation when trying to manage workload. Walk

278 elicited the lowest loading, and decreased the vertical contribution to loading, and has also been

279 associated with a reduction in pain intensity whilst riding. ${ }^{2}$

\section{Conflicts of Interest}

The authors have no conflicts of interest to declare

Acknowledgements

We wish to thank all the riders who willingly gave their time to participate in this study. Particular thanks are due to Elizabeth McCann and Fiona Hulme (Dressage coaches) for allowing access to their facility and competitors.

\section{Legend to Figures}

290 Figure 1. Placement of the GPS units at L5 and C7.

291 Figure 2. The influence of riding pace and GPS location on uni-axial PlayerLoad (a.u.).

292 Figure 3. The influence of riding pace and GPS location on relative uni-axial contributions

293 (\%) to PlayerLoad.

\section{References}


297 1. Thomas KE, Annest JL, Gilchrist J, Bixby-Hammett DM. Non-fatal horse related 298 injuries treated in emergency departments in the United States, 2001-2003. Br J Sports $299 \quad$ Med. 2006;40:619-626.

300 2. Kraft CN, Urban N, Ilg A, Wallny T, Scharfstädt A, Jäger M, Pennekamp PH. Influence of the riding discipline and riding intensity on the incidence of back pain in competitive horseback riders. Sportverletz Sportschaden. 2007;21(1):29-33.

3. Lewis V, Kennerley R. A preliminary study to investigate the prevalence of pain in elite dressage riders during competition in the United Kingdom. Comp Exerc Physiol. 2017;13(4):259-263.

4. Kraft CN, Pennekamp PH, Becker U, Young M, Diedrich O, Luring C, von Falkenhausen M. Magnetric resonance imaging findings of the lumbar spine in elite horseback riders: Correlations with back pain, body mass index, trunk/leg-length coefficient, and riding discipline. Am J Sports Med. 2009;37:2205-2213.

5. Adams MA, Hutton WC. The mechanical function of the lumbar apophyseal joints. Spine. 1983;8(3):327-330.

6. von Peinen K, Wiestner T, Bogisch S, Roepstorff L, van Weeren PR, Weishaupt MA. Relationship between the forces acting on the horse's back and the movements of rider and horse while walking on a treadmill. Equine Vet J. 2010;41:285-291.

7. Jander CB, Greene D, Mcintosh A. Profiling forces experienced by jockeys during stimulated race riding - A single case study. Med Sci Sport Exerc. 2011;43(5):426.

8. Alexander J, Hobbs SJ, May K, Northrop A, Brigden C, Selfe J. Postural characteristics of female dressage riders using 3D motion analysis and the effects of an athletic taping technique: a randomised control trial. Phys Ther Sport. 2014;16:154-161. 
320 9. Greig M, Nagy P. Tri-axial accelerometry differentiates lumbar and cervico-thoracic spine loading during cricket fast bowling. J Sport Rehabil. 2017;26(4):257-262

10. Greig M, Emmerson H, McCreadie J. Is there a role for GPS in determining functional ankle rehabilitation progression criteria? A preliminary study. J Sports Rehabil. doi: $10.1123 /$ jsr.2018-0045

11. Greig M, Child B. Sub-maximal cricket fast bowling offers a disproportionate reduction in loading vs performance: An alternative workload intervention. J Sport Rehabil. doi.org/10.1123/jsr.2018-0266

12. Fruehwirth B, Peham C, Scheidl M, Schobeberger H. Evaluation of pressure distribution under an English saddle at walk, trot and canter. Equine Vet J. 2004;36:754-757.

13. Ball D, Herrington L. Training and overload: adaptation and failure in the musculoskeletal system. J Bodywork Mov Ther. 1998;2(3):161-167.

14. Mayberry JC, Pearson TE, Wiger KJ, Diggs BS, Mullins RJ. Equestrian injury prevention efforts need more attention to novice riders. J Trauma Injury Infect Crit Care.

15. Roussouly P, Gollogly S, Berthonnaud E, Dimnet J. Classification of the normal variation in the sagittal alignment of the human lumbar spine and pelvis in the standing position. Spine. 2005;30:346-353.

16. Brodal P. Det nevrobiologiske grunnlaget for balanse (Neurological basis of balance). Fysioterapeuten. 2004;8:25-30.

340 17. Engell MT, Clayton HM, Egenvall A, Weishaupt MA, Roepstorff L. Postural changes and their effects in elite riders when actively influencing the horse versus sitting passively at trot. Comp Exerc Physiol. 2016;12(1):27-33.

18. Terada K, Clayton HM, Lanovaz JL, Kato K. Stabilization of wrist position during horseback riding at trot. Equine Comp Exerc Physiol. 2006;3:179-184. 
19. Münz F, Eckardt F, Heipertz-Hengst C, Peham C, Witte K. A preliminary study of an inertial sensor-based method for the assessment of human pelvis kinematics in dressage riding. J Equine Vet Sci. 2013;33:950-955

20. Thrasher TA, Sin VW, Mesani K, Vette AH, Craven CB, Popovik MR. Responses of the Trunk to Multidirectional Perturbations During Unsupported Sitting in Normal Adults. $J$ App Biomech. 2010;26(3):332-340.

21. Lovett T, Hodson-Tole E, Nankervis K. A preliminary investigation of rider position during walk, trot and canter. Equine Comp Exerc Physiol. 2005;2:71-76.

22. van Beek FE, de Cocq P, Timmerman E, Muller M. Stirrup forces during horse riding: a comparison between sitting and rising trot. Vet J. 2012;193(1):193-198.

23. de Cocq P, van Weeren PR, Back W. Saddle pressure measuring: Validity, reliability and power to discriminate between different saddle-fits. Vet J. 2006;172(1):265-273.

24. Panjabi MM. The stabilizing system of the spine. Part I. Function, dysfunction, adaptation, and enhancement. Spinal Disord. 1992;5(4):383-389.

25. Panjabi MM. The stabilizing system of the spine. Part II Neural zone and instability hypothesis. Spinal Disord. 1992;5:390-397.

26. McGreevy PD, Rogers LJ. Motor and sensory laterality in thoroughbred horses. Appl Anim Behav Sci. 2005;92(4):337-352.

27. Quinn S, Bird S. Influence of saddle type upon the incidence of lower back pain in equestrian riders. Br J Sports Med. 1996;30(2):140-144.

28. Murray RC, Walters J, Smart H, Dyson S, Parkin T. How do features of dressage arenas influence training surface properties which are potentially associated with lameness? Vet J. 2010;186(2):172-179. 


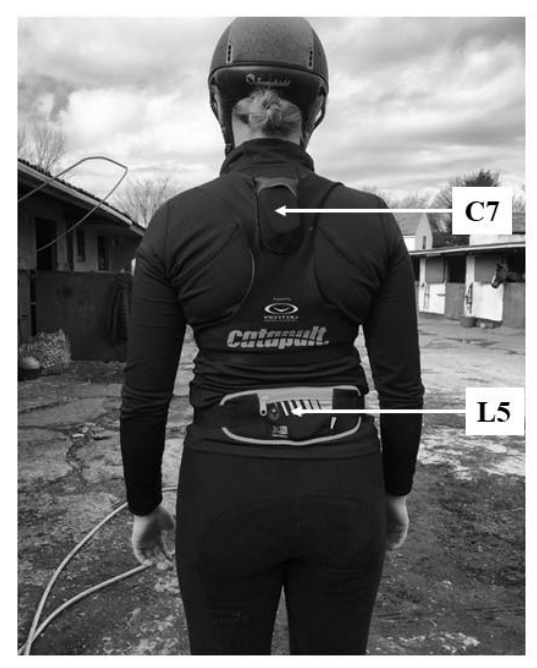

370

Figure 1. Placement of the GPS units at L5 and C7.

372

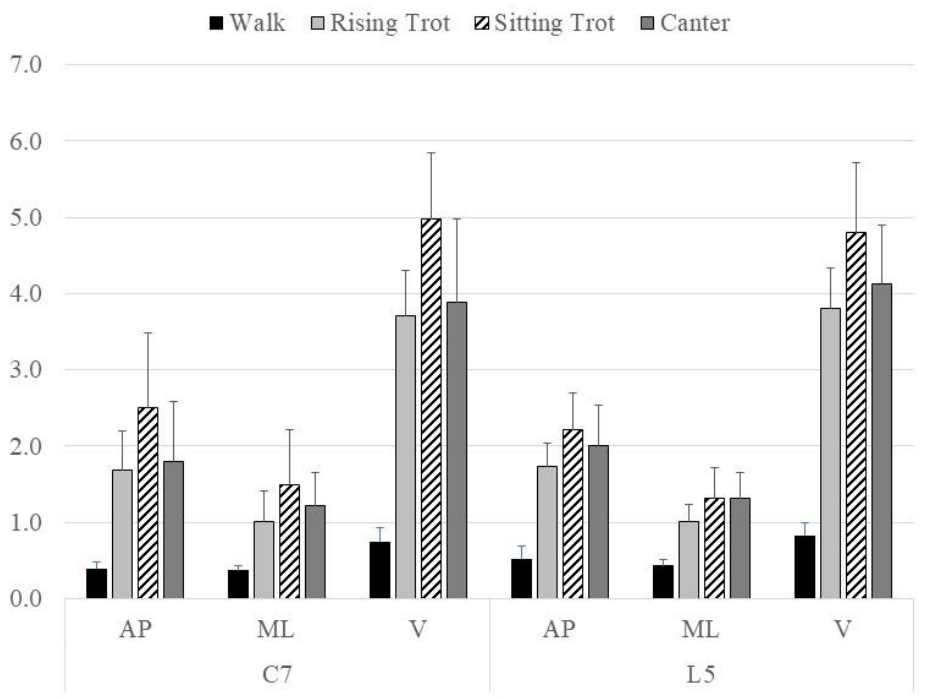

373

374 Figure 2. The influence of riding pace and GPS location on uni-axial PlayerLoad (a.u.). 


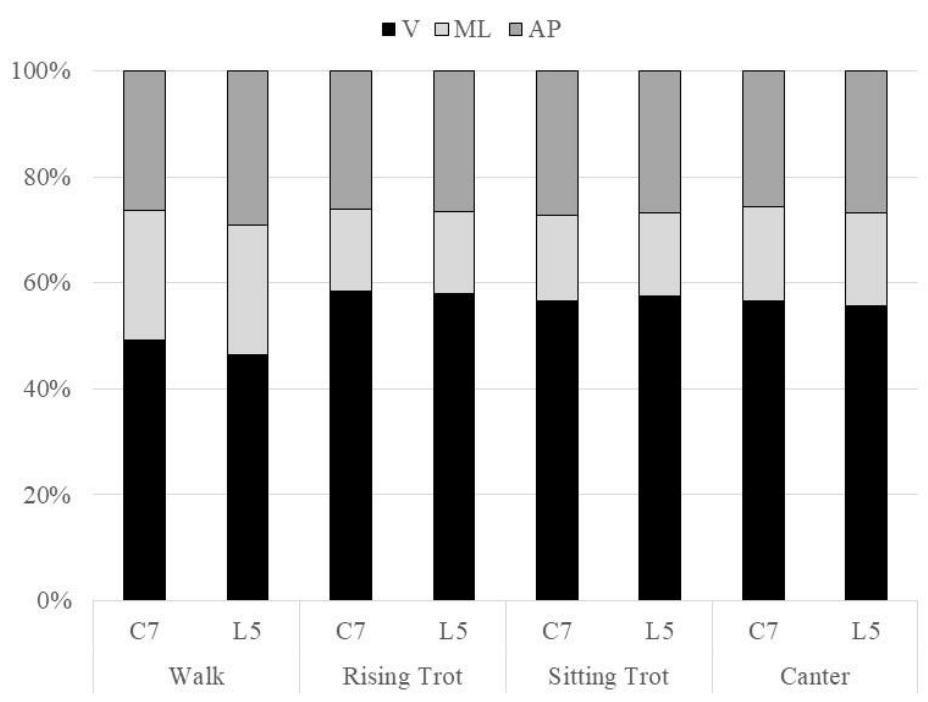

375

Figure 3. The influence of riding pace and GPS location on relative uni-axial contributions (\%) to PlayerLoad.

378 